\title{
Mensuração de feridas: revisão da literatura
}

\author{
Wound measurement: literature review
}

Thaís Dresch Eberhardta, Marciane Kesslera, Rhea Silvia de Avila Soares ${ }^{b}$, Suzinara Beatriz Soares de Limac, Graziele Gorete Portella da Fonsecad, Liane Rocha Rodriguese

a Enfermeira. Mestranda do Programa de Pós-Graduação em Enfermagem da Universidade Federal de Santa Maria (UFSM), Santa Maria, RS, Brasil.
b Enfermeira. Mestre em Enfermagem. Doutoranda do Programa de Pós-Graduação em Enfermagem da UFSM, Santa Maria, RS, Brasil.
c Enfermeira. Doutora em Enfermagem. Professora Adjunta da UFSM, Santa Maria, Brasil.
d Enfermeira. Especialista em Gestão de Organizações Públicas em Saúde. Aluna do Programa Especial de Educação da UFSM, Santa Maria, RS, Brasil.
e Acadêmica do Curso de Graduação em Enfermagem da UFSM, Santa Maria, RS, Brasil.

RESUMO Objetivo: Identificar a produção científica acerca da mensuração de feridas.

Materiais e Métodos: Revisão narrativa da literatura. A busca foi realizada nas bases de dados Literatura Latinoamericana e do Caribe em Ciências da Saúde e na Cumulative Index to Nursing \& Allied Health Literature, em junho de 2014, sendo a análise dos dados realizada a partir da proposta operativa de Minayo.

Resultados: Nove estudos foram incluídos nesta revisão, possibilitando identificar que a mensuração de feridas pode ser desenvolvida por meio de métodos invasivos ou não invasivos. Dentre os estudos selecionados, cinco relatavam sobre técnicas não invasivas, dois estudos abordaram técnicas invasivas e dois estudos relatavam sobre técnicas invasivas versus não invasivas para mensuração de feridas. As técnicas não invasivas têm sido desenvolvidas e aprimoradas com o objetivo de minimizar as limitações das técnicas invasivas de mensuração.

Conclusão: A análise dos estudos possibilitou identificar que a mensuração de feridas pode ser desenvolvida por meio de métodos invasivos como o decalque ou traçado de acetato e a mensuração por meio da régua de papel descartável; ou não invasivos como as fotografias digitais. Destaca-se que tanto as técnicas invasivas, quanto as não invasivas de mensuração, podem utilizar aferições manuais ou computacionais, sendo que maioria das pesquisas desta revisão apresentam métodos não invasivos de mensuração.

Palavras-chave: enfermagem; gestão em saúde; pesos e medidas; cicatrização; ferimentos e lesões.

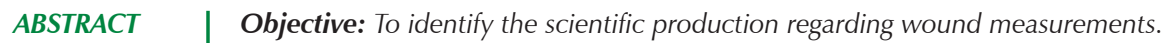

Materials and Methods: Narrative review of the literature. The search was conducted in the databases Latin American and Caribbean Health Sciences and the Cumulative Index to Nursing \& Allied Health Literature, in June 2014. Data analysis was performed based on the Minayo's operative proposal.

Results: Nine studies were included in this review, enabling to identify that the wound measurement can be developed through invasive or non-invasive methods. Among the selected studies, five reported non-invasive techniques, two studies addressed invasive techniques and two reported studies on invasive versus non-invasive wound measurement techniques. The non-invasive techniques have been developed and improved in order to minimize the limitations of invasive measurement techniques.

Conclusion: The studies identified that the wound measurement can be performed through invasive methods such as decal or acetate tracing and also through disposable paper strip; or non-invasive methods such as digital photographs. It is noteworthy that both invasive and non-invasive techniques can use manual or computer measurements, although most research of this review presented non-invasive methods of measurement.

Keywords: nursing; health management; weigths and measures; wound healing; wounds and injuries.

\footnotetext{
Correspondência:

THAís DRESCH EBERHARDT

Rua Elpídio Menezes, 21, apto. 106 - Bairro Camobi

97105-110 Santa Maria, RS, Brasil

E-mail: thaiseberhardt@gmail.com
} 


\section{INTRODUÇÃO}

A pele é o maior órgão do corpo, representa em torno de $15 \%$ do peso corporal e tem as seguintes funções: proteção, percepção do ambiente, retenção de água, termorregulação, resposta imunológica, produção de vitamina $\mathrm{D}$ e resposta emocional. Quando ocorre o rompimento da estrutura e da função anatômica normal da pele, tem-se uma ferida. Para a cicatrização destas, ocorre uma sequência de eventos complexos. Uma ferida se torna crônica quando ocorre um retardo nas etapas do processo cicatricial ${ }^{1}$.

O enfermeiro está diretamente relacionado ao cuidado a pessoas portadoras de feridas em todos os níveis de atenção à saúde. Por isso, precisa realizar uma boa avaliação clínica² . Nesta perspectiva, a avaliação das feridas é essencial para estabelecer um diagnóstico, monitorar o efeito do tratamento, identificar a presença de infecção, além de prever o resultado do tratamento com precisão ${ }^{3}$.

A partir do exposto, a mensuração apresenta-se como um dos aspectos fundamentais da avaliação de feridas, além de fornecer, de maneira objetiva e sistematizada, parâmetros que indicam a evolução da cicatrização da mesma4 .

Portanto, obter conhecimentos sobre prevenção e cuidados aos pacientes com feridas e manter-se atualizado com as evoluções da literatura científica e as recomendações com base em práticas baseadas em evidências, torna-se um desafio para os enfermeiros ${ }^{5}$, justificando o presente estudo. Para realizar a mensuração de feridas, é necessário que os enfermeiros conheçam a literatura científica sobre o assunto, a fim de tomar suas decisões baseadas em evidências.

Nesse sentido, tem-se como objetivo identificar a produção científica acerca da mensuração de feridas. Para atingi-lo, partiu-se da seguinte questão de pesquisa: "Como tem sido desenvolvida a mensuração de feridas?".

\section{MATERIAIS E MÉTODOS}

Trata-se de uma revisão narrativa da literatura. A revisão narrativa pode ser caracterizada como uma pesquisa bibliográfica ampla, sendo adequada para descrever, discutir e analisar o desenvolvimento de um determinado assunto na literatura, sob o ponto de vista teórico ou contextual ${ }^{6}$.

A busca foi realizada nas bases de dados Literatura Latinoamericana e do Caribe em Ciências da Saúde (LILACS) e Cumulative Index to Nursing \& Allied Health Literature (CINAHL), utilizando-se as estratégias indicadas na Tabela 1.

O levantamento dos estudos ocorreu no mês de junho de 2014. Utilizaram-se como critérios de inclusão: artigos originais relacionados à temática, disponíveis on-line na íntegra e gratuitamente, em inglês, português ou espanhol, sem recorte temporal. Aplicou-se como critério de exclusão os estudos sem resumo disponível nas bases de dados. Foram encontrados, inicialmente, 266 resultados, dos quais nove atenderam aos critérios de inclusão, conforme mostra a Figura 1.

Tabela 1 - Estratégias de busca realizadas e número de resultados encontrados nas bases de dados.

\begin{tabular}{|l|c|c}
\hline $\begin{array}{c}\text { Base de } \\
\text { dados }\end{array}$ & \multicolumn{1}{c}{ Estratégia } & \multicolumn{1}{c}{$\begin{array}{c}\text { Número } \\
\text { de resultados } \\
\text { encontrados }\end{array}$} \\
\hline LILACS & $\begin{array}{l}\text { ("medicao" OR "aferição" OR } \\
\text { "mensuracao" OR "medida") [Palavras] } \\
\text { AND ("ferida" OR "feridas") [Palavras] }\end{array}$ & 126 \\
& $\begin{array}{l}\text { MW"(1) (weights and measures) } \\
\text { AND MW (wounds and injuries) }\end{array}$ & 65 \\
CINAHL & $\begin{array}{l}\text { TI (2) (measure OR measurement OR } \\
\text { gauging OR mensuration) }\end{array}$ & 75 \\
\hline
\end{tabular}

MW refere-se a "Word in Subject Heading".

TI refere-se a "Title".

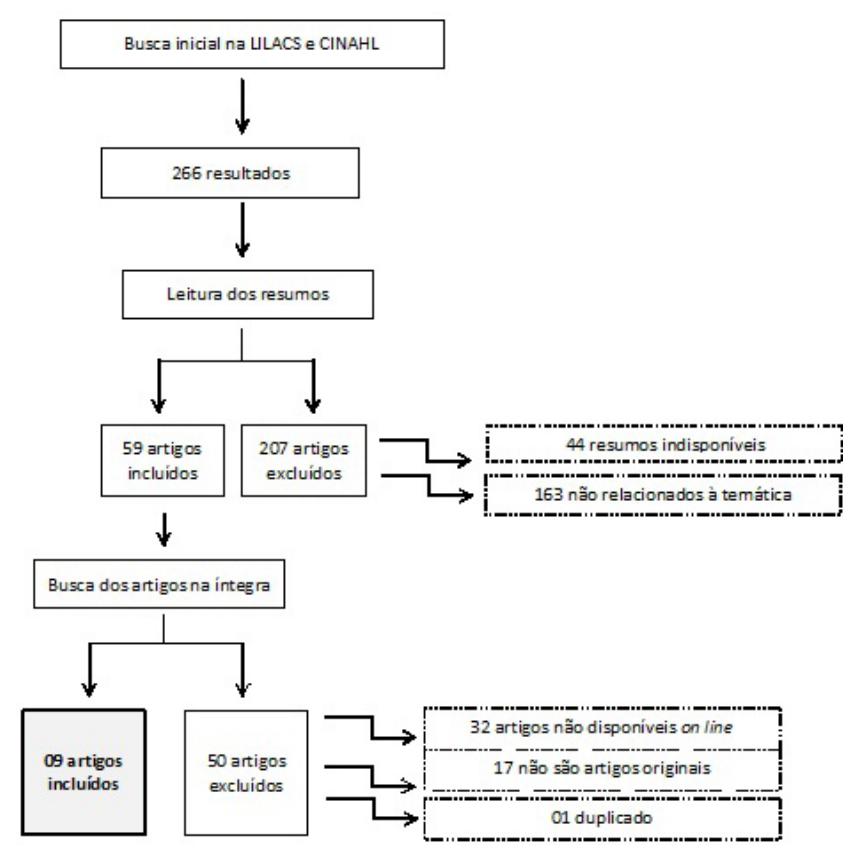

Figura 1. Fluxograma da inclusão e exclusão dos artigos no estudo de revisão (LILACS, CINAHL).

Para a descrição dos estudos selecionados, foi elaborado um quadro sinóptico, contendo as seguintes informações: identificação, referência, língua, subárea (da revista), resumo, ano de publicação, procedência (onde foi feita a pesquisa), procedência (do primeiro autor), objetivo, desenho do estudo, temática e principais resultados. A análise dos dados foi realizada qualitativamente, seguindo-se as etapas da proposta operativa de Minayo ${ }^{7}$.

Em relação às questões éticas, os preceitos quanto a autoria foram respeitados, e em virtude da natureza bibliográfica desta pesquisa, não houve necessidade de aprovação no 
Comitê de Ética em Pesquisa. Foram respeitados os preceitos da Lei que regula os direitos autorais (Lei 9.610 de 19 de fevereiro de 1998).

\section{RESULTADOS E DISCUSSÃO}

Dos nove estudos incluídos nesta revisão, quatro foram publicados em revistas da subárea de Enfermagem, três de Medicina, um de Medicina veterinária e um de Traumatologia. A maioria (seis) dos artigos foi publicada em inglês e três em português. Quanto ao ano de publicação, foi publicado um artigo nos anos de 2000, 2001 e 2009 e dois nos anos de 2006, 2010 e 2012. A Tabela 2 mostra os estudos analisados, classificados por identificação, autor, título, revista, ano e temática.

Seis estudos não informaram o local da pesquisa, uma pesquisa foi realizada no Brasil, uma na Bélgica e uma nos Estados Unidos da América. Quanto à procedência do primeiro autor, três são do Brasil, três dos Estados Unidos da América e um de cada um dos seguintes países: Bélgica, Irlanda e Irlanda do Norte.

A análise dos estudos possibilitou a identificação das seguintes categorias: técnicas não invasivas para mensuração de feridas; técnicas invasivas para mensuração de feridas e técnicas invasivas versus não invasivas para mensuração de feridas. Destacando que dos estudos selecionados, cinco relatam técnicas não invasivas; dois abordam técnicas invasivas; e dois estudos relatam sobre técnicas invasivas versus não invasivas para mensuração de feridas.

\section{Técnicas invasivas para mensuração de feridas}

Os métodos invasivos possuem limitações e desvantagens, como risco de lesão tecidual; risco de contaminação local; risco de infecção de outros pacientes ou da equipe por microorganismos presentes no exsudato ao contaminar a cama e as roupas do paciente; falhas no registro de informações a respeito da área, cor e presença de tecido de granulação ${ }^{8}$.

Em relação às técnicas invasivas para a mensuração de feridas, identifica-se, nesta revisão, o decalque ${ }^{9}$ ou traçado de acetato $^{10}$. A partir destas técnicas é possível que a mensuração seja realizada manualmente ou através de programas computacionais. O processo de reprodução e ampliação do decalque das feridas mostrou-se preciso, desde que programado em copiadora computadorizada a laser e a área mensurada através de planímetro ${ }^{9}$.

Tabela 2 - Distribuição dos estudos analisados segundo a identificação, base de dados, autor, título, revista, ano e temática (LILACS, CINAHL).

\begin{tabular}{|c|c|c|c|c|c|}
\hline ID & Autor & Título & Revista & Ano & Temática \\
\hline 1 & $\begin{array}{l}\text { Reis CLD, Cavalcante JM, } \\
\text { Rocha Júnior EFR, Neves RS, } \\
\text { Santana LA, Guadagnin RV et al. }\end{array}$ & $\begin{array}{l}\text { Mensuração de área de úlceras por } \\
\text { pressão por meio dos softwares } \\
\text { Motic e do AutoCAD }\end{array}$ & $\begin{array}{l}\text { Revista Brasileira de } \\
\text { Enfermagem }\end{array}$ & 2012 & $\begin{array}{l}\text { Técnicas não invasivas para } \\
\text { mensuração de feridas }\end{array}$ \\
\hline 2 & $\begin{array}{l}\text { Lopes AG, Soares MC, } \\
\text { Santana LA, Guadagnin RV, } \\
\text { Neves RS }\end{array}$ & $\begin{array}{l}\text { Aferição não-invasiva de úlcera } \\
\text { por pressão simulada em } \\
\text { modelo plano }\end{array}$ & $\begin{array}{l}\text { Revista Brasileira de } \\
\text { Enfermagem }\end{array}$ & 2009 & $\begin{array}{l}\text { Técnicas não invasivas para } \\
\text { mensuração de feridas }\end{array}$ \\
\hline 3 & $\begin{array}{l}\text { Falcão SC, Coelho ARB, } \\
\text { Almeida EL, Galdino APM }\end{array}$ & $\begin{array}{l}\text { Processo modificado de } \\
\text { reprodução e amplificação de } \\
\text { imagem para mensuração de área } \\
\text { por planimetria. Aplicação em } \\
\text { feridas planas produzidas em cães, } \\
\text { tratadas por curativos oclusivos de } \\
\text { pele de rã }\end{array}$ & $\begin{array}{l}\text { Brazilian Jounal Veterinary } \\
\text { Research and Animal Science }\end{array}$ & 2001 & $\begin{array}{l}\text { Técnicas invasivas para } \\
\text { mensuração de feridas }\end{array}$ \\
\hline 4 & $\begin{array}{l}\text { Poucke SV, Nelissen R, } \\
\text { Jorens P, Haeghen YV }\end{array}$ & $\begin{array}{l}\text { Comparative analysis of two } \\
\text { methods for wound bed area } \\
\text { measurement }\end{array}$ & International Wound Journal & 2010 & $\begin{array}{l}\text { Técnicas não invasivas para } \\
\text { mensuração de feridas }\end{array}$ \\
\hline 5 & $\begin{array}{l}\text { Papazoglou ES, Zubkov L, } \\
\text { Mao X, Neidrauer, Rannou N, } \\
\text { Weingarten MS }\end{array}$ & $\begin{array}{l}\text { Image analysis of chronic wounds } \\
\text { for determining the surface area }\end{array}$ & $\begin{array}{l}\text { Wound Repair and } \\
\text { Regeneration }\end{array}$ & 2010 & $\begin{array}{l}\text { Técnicas não invasivas para } \\
\text { mensuração de feridas }\end{array}$ \\
\hline 6 & Gethin G, Cowman S & $\begin{array}{l}\text { Wound measurement comparing } \\
\text { the use of acetate tracings and } \\
\text { Visitrak }^{T M} \text { digital planimetry }\end{array}$ & Journal of Clinical Nursing & 2006 & $\begin{array}{l}\text { Técnicas invasivas para } \\
\text { mensuração de feridas }\end{array}$ \\
\hline 7 & $\begin{array}{l}\text { Haghpanah S, Bogie K, } \\
\text { Wang X, Banks PG, Ho CH }\end{array}$ & $\begin{array}{l}\text { Reliability of electronic versus } \\
\text { manual wound measurement } \\
\text { techniques }\end{array}$ & $\begin{array}{l}\text { Archives of Physical Medicine } \\
\text { and Rehabilitation }\end{array}$ & 2006 & $\begin{array}{l}\text { Técnicas invasivas versus } \\
\text { não invasivas para mensuração } \\
\text { de feridas }\end{array}$ \\
\hline 8 & $\begin{array}{l}\text { Lagan KM, Dusoir AE, } \\
\text { McDonough SM, Baxter GD }\end{array}$ & $\begin{array}{l}\text { Wound measurement: the } \\
\text { comparative reliability of direct } \\
\text { versus photographic tracings } \\
\text { analyzed by planimetry versus } \\
\text { digitizing techniques }\end{array}$ & $\begin{array}{l}\text { Archives of Physical Medicine } \\
\text { and Rehabilitation }\end{array}$ & 2000 & $\begin{array}{l}\text { Técnicas invasivas versus } \\
\text { não invasivas para mensuração } \\
\text { de feridas }\end{array}$ \\
\hline 9 & Sprigle S, Nemeth M, Gajjala A & $\begin{array}{l}\text { Iterative design and testing of a } \\
\text { hand-held, non-contact wound } \\
\text { measurement device }\end{array}$ & Journal of Tissue Viability & 2012 & $\begin{array}{l}\text { Técnicas não invasivas para } \\
\text { mensuração de feridas }\end{array}$ \\
\hline
\end{tabular}


O estudo realizado por Gethin e Cowman ${ }^{10}$ comparou as mensurações de área manuais e com o software Visitrak ${ }^{\text {TM }}$ desenhadas em folhas de acetato. Os autores concluíram que a diferença entre a medida manual e do software para feridas com área $<10 \mathrm{~cm}^{2}$ não foi significativa. Já para feridas $>10 \mathrm{~cm}^{2}$ houve diferença significativa entre os dois métodos ${ }^{10}$.

\section{Técnicas não invasivas para mensuração de feridas}

As técnicas não invasivas têm sido desenvolvidas e aprimoradas com o objetivo de minimizar as limitações determinadas pelas técnicas invasivas de mensuração ${ }^{11}$. No entanto, apresentam algumas desvantagens, como alto custo, necessidade de longo período de treinamento e equipamento pesado ${ }^{8,12}$.

Em relação às técnicas não invasivas para a mensuração de feridas, foram estudadas diversas formas de análise de fotografias digitais: através do software Motic, do software AutoCAD ${ }^{\circledR 13}$, de um dispositivo de mensuração de feridas - Wound Measurement Device (WMD) - através de um smartphone comercialmente disponível ${ }^{14}$, algoritmo baseado nas diferenças de cor entre a ferida e a pele perilesional15-16. Além disso, foi estudado um sistema de projeção de pontos luminosos ${ }^{11}$.

O estudo que comparou o software AutoCAD ${ }^{\circledR}$ com o Motic concluiu que ambos apresentam pequenos erros de medida. Houve diferença estatística e encontrou-se baixa reprodutibilidade, entretanto, os programas concordam entre si e são mais confiáveis do que as técnicas simples de mensuração de área, permitindo melhor acompanhamento da evolução de feridas ${ }^{13}$.

Quando ao uso do WMD através de um smartphone comercialmente disponível, percebeu-se que este possui maior acurácia e confiabilidade do que técnicas manuais de mensuração, no entanto, possui menor acurácia e confiabilidade do que outras tecnologias baseadas em computador $^{14}$.

Ao confrontar o traçado de acetato e a mensuração da área em plataforma online, com algoritmo baseado nas diferenças de cor entre a ferida e a pele perilesional, percebeu-se que ocorreu menor variabilidade nas medições realizadas através do algoritimo. No entanto, estas variações ainda são consideráveis, sendo necessária a definição exata de bordas da ferida, separando o seu leito e a pele perilesional ${ }^{15}$.

Papazoglou et al. ${ }^{16}$ compararam a mensuração manual e através de algoritmo baseado em diferença de cores através do software MATLAB. Os autores concluíram que houve sobreposição das medidas ${ }^{16}$.
Em outro estudo, foi confeccionado um modelo plano com durepoxi, simulando uma úlcera por pressão, utilizado um sistema com projeção de pontos luminosos, realizada fotografia digital, tratamento da imagem em programa Java e posterior cálculo do volume do modelo. O método mostrou-se adequado e com erros de acurácia aceitáveis para a mensuração de profundidade e volume de feridas, porém seu uso ainda não é viável na prática clínica. Os autores sugerem que sejam realizados estudos que viabilizem o método para a prática clínica, utilizando luz ambiente ${ }^{11}$.

Outra técnica não invasiva a ser destacada se refere ao uso de fotografias como técnica não invasiva para mensuração de feridas. A utilização deste método facilita o monitoramento da cicatrização de maneira detalhada sem causar desconforto físico ao paciente, além disso, é um registro permanente de evolução da lesão. Assim, a fotografia digital consiste em uma importante ferramenta no auxílio ao cuidado de enfermagem de modo humanizado possibilitando sua utilização como base para posteriores ocorrências no mesmo contexto $^{17}$.

O registro fotográfico deve ser realizado de um ponto fixo, de maneira que as mudanças na cicatrização sejam evidenciadas, ou seja, deve-se padronizar a zona a ser fotografada, de modo a se obter o tamanho relativo da lesão, sua cor e condições da pele ao redor. Ressalta-se que os profissionais devem estar treinados para realizar estes procedimentos, e possuírem câmeras similares ${ }^{18-19}$.

\section{Técnicas invasivas versus não invasivas para mensuração de feridas}

Em relação à comparação de técnicas invasivas e não invasivas, um estudo comparou o traçado de acetato digitalizado com o registro fotográfico ${ }^{20}$ e o outro comparou a mensuração da área através da medida linear de régua com dois sistemas digitais ${ }^{21}$. Ao comparar o traçado de acetado digitalizado com o registro fotográfico, ambos com a área mensurada através de planimetria, Lagan et al. ${ }^{20}$ perceberam que o processo de digitalização possui menor variabilidade.

Haghpanah et al. ${ }^{21}$ compararam a mensuração da área através da medida linear de régua com dois sistemas digitais - Visitrak ${ }^{\text {TM }}$ e Verge Videometer $(\mathrm{VeV})$-, concluindo que as mensurações com régua possuem menor precisão do que as demais. O Visitrak ${ }^{\text {TM }}$ consiste em uma técnica de medição realizada por meio de um traçado na folha de acetato que contorna a lesão, após os dados são analisados em tablet específico, o qual irá calcular a área da lesão automaticamente. Já o VeV é um software que utiliza a 
fotografia para análise, não necessitando contato direto com a lesão ${ }^{21}$.

\section{CONSIDERAÇÕES FINAIS}

Dentre os nove estudos incluídos nesta revisão, a maioria foi publicado em revistas da subárea de Enfermagem, em inglês, nos anos de 2006, 2010 e 2012. Destaca-se que a maioria dos artigos não informou o local da pesquisa, sendo o primeiro autor procedente, na maior parte dos casos, do Brasil e Estados Unidos da América.

A análise dos estudos possibilitou identificar que a mensuração de feridas pode ser desenvolvida através de métodos invasivos como o decalque ou traçado de acetato e a mensuração através da régua de papel descartável; ou não invasivos como as fotografias digitais. Evidencia-se que tanto as técnicas invasivas, quanto as não invasivas de mensuração podem utilizar aferições manuais ou computacionais. A diferença entre os métodos está no contato direto com a lesão.

Vale ressaltar que a maioria das pesquisas desta revisão e as mais recentes apresentam métodos não invasivos para mensuração de feridas, o que pode estar relacionado à busca de alternativas que permitam realizar este processo oferecendo menores riscos ao paciente. Percebe-se, neste estudo, um quantitativo limitado de estudos voltados à mensuração de feridas, o que revela a necessidade de realizar mais pesquisas sobre a temática, especialmente com enfoque às técnicas não invasivas de mensuração. Espera-se que este estudo possa contribuir para construção de conhecimentos sobre técnicas de mensuração de feridas, compreendida como uma etapa importante na avaliação do paciente com lesão de pele, visando otimizar a assistência e o tratamento.

Ainda, espera-se contribuir para a Enfermagem como área de conhecimento, pois os resultados podem subsidiar de maneira importante as práticas de educação e assistenciais dos enfermeiros que atuam serviços de atenção a portadores de feridas e do trabalho interdisciplinar.

\section{REFERÊNCIAS}

1. Scemons D, Elston D. Nurse to nurse: cuidados com feridas. Porto Alegre: Artmed; 2011.

2. Sehnem GD, Busanello J, Silva FM, Poll MA, Borges TAP, Rocha EN. Dificuldades enfrentadas pelos enfermeiros no cuidado de enfermagem a indivíduos portadores de feridas. Ciênc cuid saude 2015;14(1):839-46. http://dx.doi.org/10.4025/cienccuidsaude. v14i1.20949

3. Keast DH, Bowering CK, Evans AW, Mackean GL, Burrows C, D'Souza L. MEASURE: a proposed assessment framework for developing best practice recommendations for wound assessment. Wound Repair Regen 2004;12:S1-17. http://dx.doi.org/10.1111/ j.1067-1927.2004.0123S1.x

4. Carvalho DV, Gomes FSL, Carmo DJAC, Batista JA, Viana MN. Planimetria como método para mensuração de feridas. Reme Rev Min Enferm 2006;10(4):425-8.

5. Ferreira AM, Rigotti MA, Barcelos LS, Simão CMF, Ferreira DN, Gonçalves RQ. Knowledge and practice of nurses about care for patients with wounds. Rev pesqui cuid fundam (Online) 2014; 6(3):1178-90. http://dx.doi.org/10.9789/2175$5361.2014 v 6 n 3 p 1178$

6. Rother ET. Revisão sistemática x revisão narrativa. Acta paul enferm 2007;20(2):v-vi. http://dx.doi.org/10.1590/S010321002007000200001

7. Minayo MCS. O desafio do conhecimento: pesquisa qualitativa em saúde. 13 ed. São Paulo: Hucitec; 2013.

8. Krouskop TA, Baker R, Wilson MS. A noncontact wound measurement system. J Rehabil Res Dev 2002;39(3):337-46.

9. Falcão SC, Coelho ARB, Almeida EL, Galdino CAPM. Processo modificado de reprodução e amplificação de imagem para mensuração de área por planimetria: Aplicação em feridas planas produzidas em cães, tratadas por curativos oclusivos de pele de rã Braz j vet res anim sci 2001;38(4):165-9. http://dx.doi.org/10.1590/ S1413-95962001000400004

10. Gethin G, Cowman S. Wound measurement comparing the use of acetate tracings and Visitrak digital planimetry. J Clin Nurs 2006;15(4):422-7. http://dx.doi.org/10.1111/j.1365-2702.2006. 01364.x

11. Lopes AG, Soares MC, Santana LA, Guadagnin RV, Neves RS. Aferição não-invasiva de úlcera por pressão simulada em modelo plano. Rev Bras Enferm 2009;62(2):200-3. http://dx.doi.org/10.1590/S003471672009000200005

12. Ministério da Saúde. Secretaria de Políticas de Saúde. Departamento de Atenção Básica. Área Técnica de Dermatologia Sanitária. Manual de condutas para úlceras neurotróficas e traumáticas. Brasília: Ministério da Saúde; 2002.

13. Reis CLD, Cavalcante JM, Rocha Júnior EFR, Neves RS, Santana LA, Guadagnin RV, Brasil LM. Mensuração de área de úlceras por pressão por meio dos softwares Motic e do AutoCAD ${ }^{\circledR}$. Rev Bras Enferm 2012;65(2):304-8. http://dx.doi.org/10.1590/S003471672012000200016

14. Sprigle S, Nemeth M, Gajjala A. Iterative design and testing of a hand-held, non-contact wound measurement device. J Tissue Viability 2012;21(1):17-26. http://dx.doi.org/10.1016/j.jtv.2011. 09.001

15. Poucke SV, Nelissen R, Jorens P, Haeghen YV. Comparative analysis of two methods for wound bed area measurement. Int Wound J 2010;7(5):366-77. http://dx.doi.org/10.1111/j.1742481X.2010.00701.x

16. Papazoglou ES, Zubkov L, Mao X, Neidrauer M, Rannou N, Weingarten MS. Image analysis of chronic wounds for determining the surface area. Wound Repair Regen 2010;18(4):349-58. http://dx.doi.org/10.1111/j.1524-475X.2010.00594.x 
17. Galvão MTG, Alexandre HO, Dantas PB, Lima ICV, Lopes EM. Uso da fotografia no processo do cuidar: tendências das ações de enfermagem. Cienc enferm 2013;19(3);31-9.

18. Cooper DM. Assessment, measurement, and evaluation: their pivotal roles in wound healing. In: Bryant RA. Acute and chronic wounds: nursing management. 2rd ed. St. Louis: Mosby; 2000. p. 51-83.

19. Baumgarten M, Margolis DJ, Selekof JL, Moye N, Jones PS, Shardell M. Validity of pressure ulcer diagnosis using digital photography. Wound Repair Regen 2009;17(2):287-90. http://dx.doi.org/ 10.1111/j.1524-475X.2009.00462.x
20. Lagan KM, Dusoir AE, McDonough SM, Baxter GD. Wound measurement: the comparative reliability of direct versus photographic tracings analyzed by planimetry versus digitizing techniques. Arch Phys Med Rehabil 2000;81(8):1110-6. http:// dx.doi.org/10.1053/apmr.2000.6281

21. Haghpanah S, Bogie K, Wang X, Banks PG, Ho CH. Reliability of electronic versus manual wound measurement techniques. Arch Phys Med Rehabil 2006;87(10):1396-402. http://dx.doi. org/10.1016/j.apmr.2006.06.014 